\title{
Prevalence, symptomatic features, and factors associated with sleep disturbance/insomnia in Japanese patients with type-2 diabetes
}

This article was published in the following Dove Press journal:

Neuropsychiatric Disease and Treatment

18 July 2017

Number of times this article has been viewed

\author{
Hajime Narisawa' \\ Yoko Komada' \\ Takashi Miwa² \\ Junpei Shikuma² \\ Mamoru Sakurai \\ Masato Odawara ${ }^{2}$ \\ Yuichi Inoue ${ }^{1,3}$ \\ 'Department of Somnology, \\ ${ }^{2}$ Department of Diabetes, \\ Endocrinology and Metabolism, Tokyo \\ Medical University, Shinjuku-ku, \\ 3Japan Somnology Center, Institute \\ of Neuropsychiatry, Shibuya-ku, \\ Tokyo, Japan
}

Correspondence: Yuichi Inoue Japan Somnology Center, Institute of Neuropsychiatry, I-24-10 Yoyogi, Shibuya-ku, Tokyo I5I0053, Japan

$\mathrm{Tel}+81333749112$

Fax +8I 333749125

Email inoue@somnology.com
Purpose: To clarify the prevalence and symptomatic characteristics of sleep disturbance/ insomnia among type-2 diabetes mellitus (DM) Japanese patients.

Methods: A cross-sectional survey of Japanese patients with the disorder was conducted. Participants consisted of 622 type-2 DM patients (mean 56.1 \pm 9.56 years) and 622 sex- and agematched controls. Participants' scores in the Japanese version of the Pittsburgh Sleep Quality Index (PSQI-J), the Japanese version of the 12-item Center for Epidemiologic Studies Depression Scale (CES-D), the Medical Outcomes Study 8-item Short Form Health Survey (SF-8), and the glycated hemoglobin A1c (HbA1c) of type-2 DM patients were analyzed.

Results: There were 253 poor sleepers (43.9\%) in the type-2 DM group as a result of dichotomization with the PSQI-J cutoff total score of 5.5. The type-2 DM group recorded a higher mean PSQI-J total score $(P<0.01)$ and manifested poorer sleep maintenance. Poor sleepers in both groups had lower mental component summary from SF-8 (MCS), physical component summary from SF-8 (PCS), and CES-D than good sleepers, and good sleepers in both groups had higher MCS, PCS, and CES-D than poor sleepers. Higher body mass index, presence of smoking habit, and living alone were significantly associated with sleep disturbance/insomnia symptoms, but $\mathrm{HbAlc}$ was not associated with sleep disturbance/insomnia in the type-2 DM group.

Conclusion: Individuals affected with type-2 DM are likely to experience sleep problems, characterized by disturbance in sleep maintenance. Sleep disturbance/insomnia symptoms in DM patients might considerably reduce health-related quality of life.

Keywords: cross-sectional survey, depression, glycated hemoglobin A1c, quality of life, subjective sleep quality

\section{Introduction}

Type-2 diabetes mellitus (DM) is characterized by a chronic condition of beta-cell dysfunction against a background of obesity-related insulin resistance. The disorder is well known to be associated with an increased risk of mortality as well as placing a substantial economic burden on public health care budget. ${ }^{1}$ Type-2 DM has a significant and consistent association with depressive symptoms and various somatic complications such as neuropathy, retinopathy, nephropathy, and cardiovascular diseases. $^{2}$ Thus, the high prevalence of type-2 DM has become a global issue as a lifestyle-related disease that may increase the risk of serious physical and psychological health issues.

Approximately $37 \%-50 \%$ of type- 2 DM patients have sleep problems, and the rate of individuals having insomnia is higher than the general population. ${ }^{3-6}$ Reduced sleep quality with low levels of slow-wave sleep, as occurs in many obese individuals, may 
contribute to increase the risk of type- 2 DM. ${ }^{5}$ Both sleep difficulties and poor sleep quality are thought to worsen diabetes symptoms. A prospective study has suggested that type-2 DM individuals may be at risk of developing insomnia, ${ }^{7}$ and a meta-analysis has found that poor quality and quantity of sleep were consistently and significantly associated with an elevated risk of developing type-2 DM. ${ }^{4,8}$ Studies of sleep deprivation have indicated that disturbed or reduced sleep impairs glucose tolerance and increases the risk of developing type-2 DM. ${ }^{9-14}$ Thus, it is possible that type-2 DM and insomnia symptoms intensify each other.

To date, numerous epidemiological studies on sleep disturbance in type-2 DM patients have been conducted in Western countries. ${ }^{15-19}$ On the other hand, it has been reported that those who had sleep disturbances showed two- to threefold higher risk of onset of type-2 DM by an 8-year prospective study investigating the relationship between sleep disturbance and subsequent onset of type- 2 DM among Japanese men. ${ }^{20}$ Sleep disturbance and depressive symptoms correlated with the health-related quality of life (HRQOL) of Japanese patients with type-2 DM. ${ }^{21}$ It has been already known that the pathophysiology of Japanese type-2 DM is different from that of Caucasian in the sense that Japanese are unable to compensate insulin resistance although insulin secretion is increased to almost the same extent as Caucasian. ${ }^{15}$ Detailed inclusive information about the prevalence of sleep disturbance/insomnia among Japanese type-2 DM patients and the impact and characteristics of sleep disturbance/insomnia symptoms in this population as compared with normal population including both sex have not been ascertained. Taking these issues into consideration, we conducted a cross-sectional study on Japanese type-2 DM patients focusing on the content of insomnia symptom.

\section{Methods}

\section{Participants and procedures}

This study was approved by the ethics committee of Tokyo Medical University, Japan, and written informed consent was obtained from all participants.

Attending physicians invited consecutive type-2 DM patients who visited the outpatient clinic for diabetes, endocrinology, and metabolism consultations at Tokyo Medical University Hospital, Japan, in February 2014 to fill in the questionnaire. A total of 881 patients out of 1,092 agreed to participate in the survey and completed the questionnaire described in the following paragraph with a response rate of $80.7 \%$. Blood samples from these patients were collected on the same day for glycated hemoglobin A1c (HbA1c) analysis. We excluded 256 participants whose age was outside the age range of 30-69 years, to align with the general population group as indicated below, and three participants who failed to complete the items for sex or age. This process yielded 622 participants (472 males, 150 females; mean $56.1 \pm 9.56$ years) who constituted the type-2 DM group. Of these, 464 patients were taking oral hypoglycemic agent, and 169 patients were receiving insulin therapy. The general population group of 622 participants was randomly sampled from participants in our cohort survey that was conducted for dietary assessment research in 2014 ( $n=2,007$; age range 30-69 years). The general population group was matched for sex and age with the type-2 DM group. All the subjects in the type-2 DM group and the general population group were not engaged in shift work at the investigation.

Questionnaires used for this survey consisted of the following: the Japanese version of the Pittsburgh Sleep Quality Index (PSQI-J), the Japanese version of the 12-item Center for Epidemiologic Studies Depression Scale (CES-D), and the 8-item Short Form Health Survey of the Medical Outcomes Study (SF-8). Demographic variables including age, sex, body mass index (BMI), living alone or with others, family members needing in-home nursing care, and regular employment were collected. Information was also gathered about personal behaviors such as habitual smoking, alcohol consumption, and physical exercise.

Subjective sleep difficulty was assessed with the validated PSQI-J, ${ }^{22}$ translated from the original version. ${ }^{23}$ The PSQI-J is a self-rated questionnaire that measures sleep difficulty including sleep disturbance retrospectively for a 1-month period, with total scores ranging from 0 to 21 . Higher PSQI-J scores indicate more severe sleep difficulty, and a total PSQI-J score $>5.5$ yields a diagnostic sensitivity of $85.7 \%$ for pathological insomnia. ${ }^{22}$ The questionnaire measures seven subitems for sleep difficulty (C1: sleep quality; C2: sleep-onset latency; $\mathrm{C} 3$ : sleep duration; $\mathrm{C} 4$ : sleep efficiency; C5: sleep disturbance; C6: use of sleep medications; C7: daytime dysfunction). This scale has been used for measuring sleep disturbance/insomnia in previous epidemiological studies. ${ }^{24}$

The Japanese 12-item version of the CES-D was used to assess depressive symptoms. ${ }^{25,26}$ The total score of the CES-D has a range of $0-36$ points and participants with scores $>12$ points are defined as having depressive symptoms. ${ }^{26} \mathrm{We}$ also used the SF-8, which is a simpler version of SF-36 that is as useful for evaluating HRQOL. ${ }^{27-29}$ The SF-8, which measures the domains of vitality, social functioning, mental health, 
performance in physical role, performance in emotional role, general health perceptions, physical functioning, and bodily pain, was calculated according to the standard method. ${ }^{27}$ The physical component summary from SF-8 (PCS) and the mental component summary from SF-8 (MCS) were employed as indices of physical and mental quality of life, respectively. The general population averages for the scores were set at 50 points. Therefore, a subject with a score of $<50$ points was considered to have a diminished HRQOL. ${ }^{28}$

\section{Data analysis}

A one-way analysis of variance (ANOVA) was used for the comparisons of clinical descriptive variables between the general population and type-2 DM groups. ANOVA was also used for the comparisons among four subgroups divided by good and poor sleepers and by the type-2 DM group and the general population group, respectively. We used the chi-square test to compare categorical variables between the general population and type-2 DM groups.

In the present study, factors associated with poor sleepers in the type-2 DM group were investigated, using a series of logistic regression analyses with independent variables of age, sex, BMI, HbAlc, presence/absence of smoking, habitual alcohol consumption, living alone, family members requiring home-based nursing care, exercise habits, and regular employment. All variables were investigated initially in univariate models. We performed multivariate logistic regression analyses for all variables that showed a significant association in univariate models in order to control for confounding factors and to determine the primary correlates. Statistical tests of the regression estimates' odds ratios (ORs) were based on Wald statistics. ORs and their $95 \%$ confidence intervals (CIs) are presented to show the association.

A value of $P<0.05$ was considered significant. SPSS (11.5 for Windows; Tokyo, Japan) was used for all statistical analyses.

\section{Results}

\section{Descriptive information about study participants}

The demographic characteristics of the participants are presented in Table 1. Both the general population and type-2 DM groups included 472 males and 150 females, and the mean (standard deviation [SD]) ages of the two groups were 56.1 (9.56) years and 56.8 (9.62) years (range; 30-69 years), respectively; there was no significant difference between the groups $(F(1,1,242)=1.83$, ns $)$. The mean BMI of the type-2 DM group was significantly higher than the general population group $(F(1,1,239)=144.4, P<0.01)$. There was a significantly smaller number of individuals who habitually consumed alcohol and who were regularly employed in the type-2 DM group. The type-2 DM group contained a significantly greater number of individuals with family members who needed home-based nursing care and who lived alone $\left(\chi^{2}(1)=33.8, P<0.01 ; \chi^{2}(1)=5.73, P<0.01 ; \chi^{2}(1)=4.41\right.$, $P<0.05 ; \chi^{2}(1)=7.83, P<0.01$, respectively).

\section{Comparisons of subjective sleep evaluation and HRQOL among the subject groups}

As shown in Table 2, there were significant differences in total score and some subitem scores of PSQI-J between the two groups. ANOVA found that the type-2 DM group had worse total scores for PSQI-J, C3 (sleep duration), C4 (sleep efficiency), and C6 (use of sleep medication) than the general population group $(F(1,1,206)=7.16, P<0.01$; $F(1,1,242)=7.17, P<0.01 ; F(1,1,227)=19.3, P<0.01$; $F(1,1,239)=4.19, P<0.05$, respectively). On the other hand, $\mathrm{C} 1$ (sleep quality), C2 (sleep-onset latency), C5 (sleep disturbance), and C7 (daytime dysfunction) were not significantly different between the two groups $(F(1,1,235)=0.03$, ns; $F(1,1,235)=2.37$, ns; $F(1,1,242)=2.55$, ns; $F(1,1,238)=0.35$, $\mathrm{ns}$, respectively). The scores for each item in $\mathrm{C} 5$, which records the reasons for disturbed sleep, were not significantly different between the two groups. The $\mathrm{C} 5$ reasons are as follows: 1) cannot get to sleep within 30 minutes, 2) wake up in the middle of the night or early morning, 3) have to get up to use the bathroom, 4) cannot breathe comfortably, 5) cough or snore loudly, 6) feel too cold, 7) feel too hot, 8) have bad dreams, and 9) experience pain $(F(1,1,237)=3.10$, ns; $F(1$, $1,239)=1.61$, ns; $F(1,1,240)=0.38$, ns; $F(1,1,240)=0.42$, $\mathrm{ns} ; F(1,1,237)=2.38$, ns; $F(1,1,242)=0.21$, ns; $F(1$, $1,239)=3.17$, ns; $F(1,1,241)=0.75$, ns; $F(1,1,241)=0.33$, ns, respectively).

In the type- 2 DM group, there were 253 poor sleepers (43.9\%) as a result of dichotomization with the PSQI-J cutoff point of 5.5 for the total score. This was a higher proportion than the general population group, but not significantly so $\left(38.4 \% ; \chi^{2}(1)=3.68, P=0.06\right)$. The prevalence of sleep disturbance/insomnia symptom was not significantly different between male and female in the type-2 DM group (males: $43.2 \%$, females: $\left.45.8 \% ; \chi^{2}(1)=0.30, \mathrm{~ns}\right)$, although females showed higher prevalence in the general population group (males: $35.6 \%$, females: $47.3 \%$; $\chi^{2}(1)=6.63, P<0.05$ ).

The comparison of PCS, MCS, and CES-D among the four groups divided by group (DM/general population) and 
Table I Comparison of demographic data between control and type-2 DM group

\begin{tabular}{|c|c|c|c|c|c|c|c|}
\hline \multirow[t]{2}{*}{ Factors } & \multicolumn{2}{|c|}{ Control $(n=622)$} & \multicolumn{2}{|c|}{ Type-2 DM $(\mathrm{n}=622)$} & \multirow[t]{2}{*}{$F / X^{2}$} & \multirow[t]{2}{*}{$P$-value } & \multirow[t]{2}{*}{$\omega^{2} / V$} \\
\hline & Mean & SD & Mean & SD & & & \\
\hline Age & 56.1 & 9.56 & 56.8 & 9.62 & $\mathrm{I} .83$ & 0.18 & 0.00 \\
\hline BMI & 23.0 & 3.09 & 25.7 & 4.75 & $144.42 * *$ & 0.00 & 0.10 \\
\hline PCS & 48.4 & 6.49 & 47.1 & 7.65 & $9.55^{* *}$ & 0.00 & 0.01 \\
\hline MCS & 49.2 & 7.38 & 49.0 & 7.30 & 0.18 & 0.67 & 0.00 \\
\hline CES-D & 4.14 & 5.66 & 4.08 & 5.76 & 0.04 & 0.84 & 0.00 \\
\hline Sex & & & & & 0.00 & 1.00 & 0.00 \\
\hline Male & \multicolumn{2}{|c|}{$472(75.9 \%)$} & \multicolumn{2}{|c|}{472 (75.9\%) } & & & \\
\hline Female & \multicolumn{2}{|c|}{$150(24.1 \%)$} & \multicolumn{2}{|c|}{$150(24.1 \%)$} & & & \\
\hline Smoking habit & & & & & 0.13 & 0.75 & 0.00 \\
\hline Yes & \multicolumn{2}{|c|}{159 (25.6\%) } & \multicolumn{2}{|c|}{164 (26.5\%) } & & & \\
\hline No & \multicolumn{2}{|c|}{463 (74.4\%) } & \multicolumn{2}{|c|}{456 (73.5\%) } & & & \\
\hline Habitual alcohol consumption & & & & & $33.81 * *$ & 0.00 & 0.17 \\
\hline Yes & \multicolumn{2}{|c|}{$365(58.7 \%)$} & \multicolumn{2}{|c|}{$262(42.2 \%)$} & & & \\
\hline No & \multicolumn{2}{|c|}{257 (4I.3\%) } & \multicolumn{2}{|c|}{359 (57.8\%) } & & & \\
\hline Living alone & & & & & $7.83 *$ & 0.01 & 0.08 \\
\hline Yes & \multicolumn{2}{|c|}{108 (17.4\%) } & \multicolumn{2}{|c|}{147 (23.8\%) } & & & \\
\hline No & \multicolumn{2}{|c|}{$514(82.6 \%)$} & \multicolumn{2}{|c|}{$47 \mathrm{I}(76.2 \%)$} & & & \\
\hline Home-based nursing care & & & & & $4.4 I^{*}$ & 0.05 & 0.06 \\
\hline Yes & \multicolumn{2}{|c|}{$35(5.6 \%)$} & \multicolumn{2}{|c|}{$52(8.7 \%)$} & & & \\
\hline No & \multicolumn{2}{|c|}{$586(94.4 \%)$} & \multicolumn{2}{|c|}{$543(91.3 \%)$} & & & \\
\hline Exercise habit & & & & & 2.22 & 0.14 & 0.04 \\
\hline Yes & \multicolumn{2}{|c|}{$250(40.2 \%)$} & \multicolumn{2}{|c|}{$223(36.1 \%)$} & & & \\
\hline No & \multicolumn{2}{|c|}{$372(59.8 \%)$} & \multicolumn{2}{|c|}{$395(63.9 \%)$} & & & \\
\hline Occupation & & & & & $5.73 *$ & 0.02 & 0.08 \\
\hline Yes & \multicolumn{2}{|c|}{289 (83.0\%) } & $458(76$ & & & & \\
\hline No & $59(17$. & & $|4|(23$ & & & & \\
\hline
\end{tabular}

Notes: Continuous variables are presented as the mean and SD, while categorical variables are presented as number of participants and its percentage. Analysis of variance was used for the comparisons of clinical descriptive variables between control and diabetes groups, whereas chi-square test was conducted for categorical variables. $* P<0.05, * * P<0.01$.

Abbreviations: DM, diabetes mellitus; SD, standard deviation; BMI, body mass index; PCS, physical component summary from SF-8; MCS, mental component summary from SF-8; CES-D, Center for Epidemiological Studies Depression Scale.

the PSQI-J total score (cutoff point 5.5) found a significant difference in each score $(F(3,1,194)=32.4, P<0.01$; $F(3,1,194)=78.4, P<0.01 ; F(3,1,193)=130.0, P<0.01$, respectively). Bonferroni's post hoc analysis found no significant difference in the PCS, MCS, and CES-D score between good sleepers in the type-2 DM group and good sleepers in the general population groups. However, poor sleepers in the type-2 DM group had lower PCS than poor sleepers in the general population group, whereas MCS and CES-D were not significantly different between the poor sleepers in the two groups. Good sleepers in the general population group and those in the type-2 DM group had significantly higher MCS

Table 2 Comparison of subitems of PSQI between control and type-2 DM group

\begin{tabular}{|c|c|c|c|c|c|c|c|}
\hline \multirow[t]{2}{*}{ Factors } & \multicolumn{2}{|c|}{ Control $(n=622)$} & \multicolumn{2}{|c|}{ Type-2 DM (n=622) } & \multirow[t]{2}{*}{$\boldsymbol{F}$} & \multirow[t]{2}{*}{$P$-value } & \multirow[t]{2}{*}{$\omega^{2}$} \\
\hline & Mean & SD & Mean & SD & & & \\
\hline PSQI total score & 5.23 & 2.93 & 5.70 & 3.17 & $7.16 * *$ & 0.01 & 0.01 \\
\hline CI (sleep quality) & 1.23 & 0.66 & 1.22 & 0.71 & 0.03 & 0.85 & 0.00 \\
\hline C2 (sleep-onset latency) & 0.79 & 0.86 & 0.87 & 0.94 & 2.37 & 0.12 & 0.00 \\
\hline C3 (sleep duration) & 1.28 & 0.84 & 1.41 & 0.89 & $7.17^{* *}$ & 0.01 & 0.00 \\
\hline C4 (sleep efficiency) & 0.16 & 0.52 & 0.32 & 0.76 & $19.27 * *$ & 0.00 & 0.01 \\
\hline C5 (sleep disturbance) & 0.87 & 0.47 & 0.91 & 0.52 & 2.55 & 0.11 & 0.00 \\
\hline C6 (use of sleep medications) & 0.24 & 0.77 & 0.34 & 0.92 & $4.19 *$ & 0.04 & 0.00 \\
\hline C7 (daytime dysfunction) & 0.66 & 0.79 & 0.69 & 0.82 & 0.35 & 0.56 & 0.00 \\
\hline
\end{tabular}

Note: $* P<0.05, * * P<0.01$.

Abbreviations: PSQI, Pittsburgh Sleep Quality Index; DM, diabetes mellitus; SD, standard deviation. 

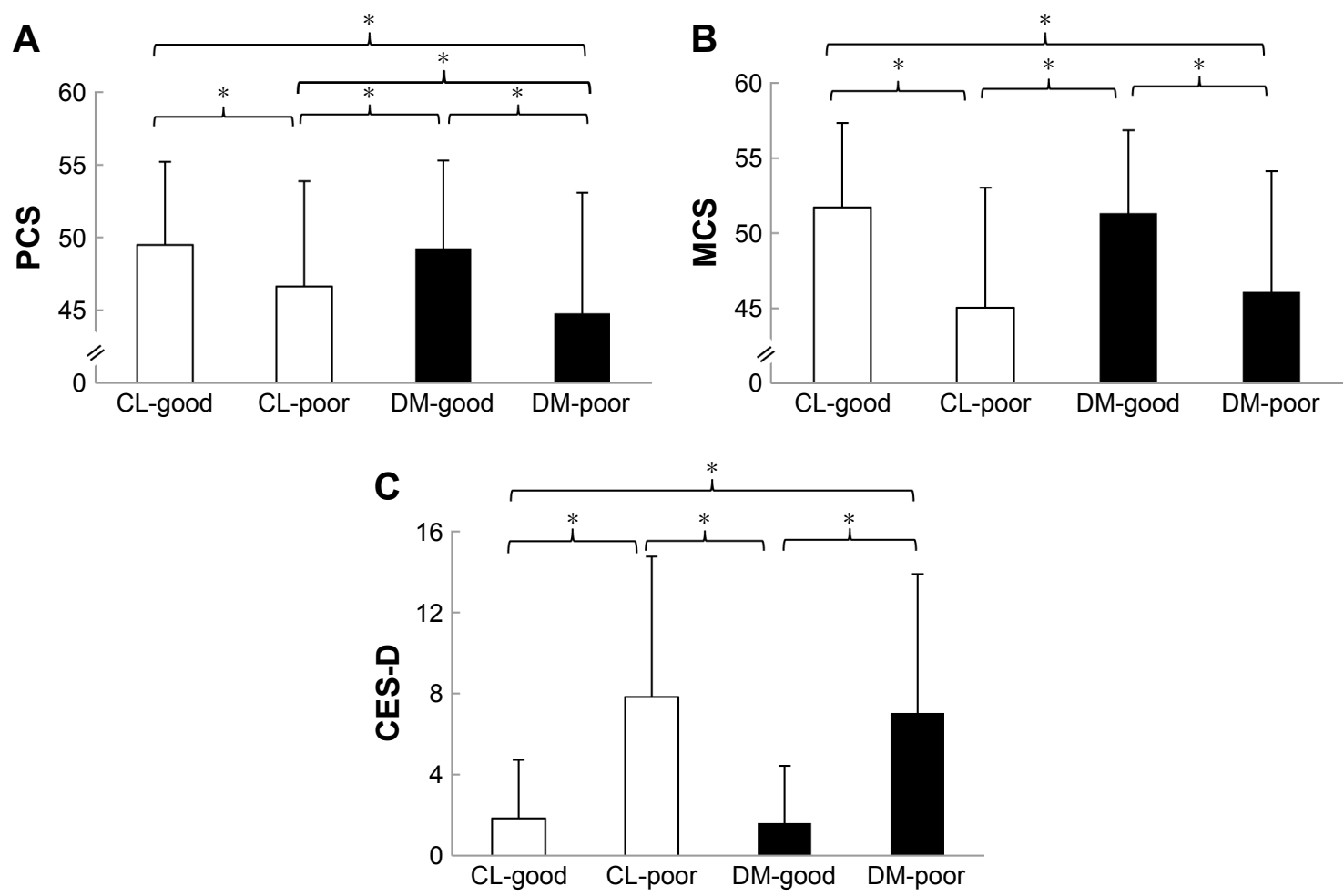

Figure I Comparison of PCS (A), MCS (B), and CES-D (C) scores among four groups categorized by groups and by the cutoff point (5.5) of PSQI total score. Notes: Error bars indicate standard deviations. ${ }^{*} P<0.05$. CL-good, good sleepers in the control group; CL-poor, poor sleepers in the control group; DM-good, good sleepers in the type-2 DM group; DM-poor, poor sleepers in the type-2 DM group.

Abbreviations: PCS, physical component summary from SF-8; MCS, mental component summary from SF-8; CES-D, Center for Epidemiologic Studies Depression Scale; PSQI, Pittsburgh Sleep Quality Index.

than poor sleepers in the respective groups. Moreover, poor sleepers in the general population group and the type-2 DM group had higher CES-D than good sleepers in the general population group and the type-2 DM group (Figure 1).

\section{Factors associated with sleep disturbance/insomnia in subjects with type-2 DM}

The factors associated with sleep disturbance/insomnia (PSQI-J score over 5.5) were investigated using univariate and multivariate logistic regression analyses for the type-2 DM group (Table 3). Univariate logistic analysis found that five independent variables were significant risk factors for sleep disturbance/insomnia in the type-2 DM group: being younger, having a higher BMI, habitual smoking, living alone, and a lack of habitual exercise. Multivariate logistic analysis with these significant variables found sleep disturbance/insomnia in this group was significantly associated with higher BMI (OR=1.05, 95\% CI: 1.01-1.09), habitual smoking $(\mathrm{OR}=1.55,95 \% \mathrm{CI}$ : $1.05-2.28)$, and living alone (OR=2.22, 95\% CI: 1.49-3.32). On the other hand, the value of HbA1c was not a significant risk factor for sleep disturbance/insomnia in the type-2 DM group.

\section{Discussion}

The purpose of this study was to investigate systematically the prevalence, symptomatic features, impact on daytime functioning, and factors associated with sleep disturbance/ insomnia in Japanese type-2 DM patients and compare the results with an age- and sex-matched Japanese general population group. There were differences and similarities between the two groups. For individuals with type-2 DM, the mean total PSQI-J score and three of seven subitems were significantly higher than those for the general population group. The proportion of individuals with sleep disturbance/insomnia was not significantly different between the groups. The reason of this result may include wide age range of the study participants, difference in medication, and a lack of health screening especially in the general population group. Thus, in line with previous studies on Western populations, ${ }^{16}$ sleep disturbance/insomnia symptoms may be more prevalent in type-2 DM individuals in the Japanese population, although the difference may not always be significant. Sleep disturbance/insomnia is generally found more common in female, however, female preponderance in insomnia seemed to be lost in the type-2 DM group. Of note, the type-2 DM group had significantly higher scores 
Table 3 Univariate and multivariate logistic regression results for factors associated with insomnia among type-2 DM

\begin{tabular}{|c|c|c|c|c|c|c|c|}
\hline \multirow[t]{2}{*}{ Factors } & \multirow{2}{*}{$\begin{array}{l}\text { Total } \\
\text { n }\end{array}$} & \multicolumn{2}{|c|}{ Insomniacs } & \multirow{2}{*}{$\begin{array}{l}\text { Univariate } \\
\text { relative risk } \\
(95 \% \mathrm{CI})\end{array}$} & \multirow[t]{2}{*}{$P$-value } & \multirow{2}{*}{$\begin{array}{l}\text { Multivariate } \\
\text { relative risk } \\
(95 \% \mathrm{CI})\end{array}$} & \multirow[t]{2}{*}{$P$-value } \\
\hline & & $\bar{n}$ & $\%$ & & & & \\
\hline \multicolumn{8}{|l|}{ Sex } \\
\hline Male & 442 & 191 & 43.2 & & ns & & ns \\
\hline Female & 144 & 66 & 45.8 & & & & \\
\hline Age & 586 & 257 & 43.9 & $0.97(0.96-0.99)$ & 0.00 & & ns \\
\hline $\mathrm{BMI}$ & 583 & 257 & 44.1 & $1.06(1.02-1.10)$ & 0.00 & $1.05(1.01-1.09)$ & 0.02 \\
\hline $\mathrm{HbAlc}$ & 576 & 253 & 43.9 & & ns & & ns \\
\hline \multicolumn{8}{|c|}{ Smoking habit } \\
\hline Yes & 152 & 80 & 52.6 & $1.63(1.13-2.37)$ & 0.01 & $1.55(1.05-2.28)$ & 0.03 \\
\hline No & 432 & 175 & 40.5 & & & & \\
\hline \multicolumn{8}{|c|}{ Habitual alcohol consumption } \\
\hline Yes & $24 I$ & 98 & 40.7 & & ns & & ns \\
\hline No & 344 & 158 & 45.9 & & & & \\
\hline \multicolumn{8}{|c|}{ Living alone } \\
\hline Yes & 139 & 83 & 59.7 & $2.32(1.57-3.43)$ & 0.00 & $2.22(1.49-3.32)$ & 0.00 \\
\hline No & 444 & 173 & 39.0 & & & & \\
\hline \multicolumn{8}{|c|}{ Home-based nursing care } \\
\hline Yes & 47 & 25 & 53.2 & & ns & & ns \\
\hline No & 514 & 219 & 42.6 & & & & \\
\hline \multicolumn{8}{|c|}{ Exercise habit } \\
\hline Yes & 209 & 79 & 37.8 & $0.68(0.48-0.96)$ & 0.03 & & ns \\
\hline No & 374 & 177 & 47.3 & & & & \\
\hline \multicolumn{8}{|c|}{ Occupation } \\
\hline Yes & 429 & 191 & 44.5 & & ns & & ns \\
\hline No & 135 & 55 & 40.7 & & & & \\
\hline
\end{tabular}

Note: The cutoff point of PSQI-J was 5.5 .

Abbreviations: DM, diabetes mellitus; Cl, confidence interval; ns, not significant; BMI, body mass index; HbAlc, glycated hemoglobin Alc; PSQI, Pittsburgh Sleep Quality Index.

for difficulty with sleep duration and sleep efficiency than the general population group, suggesting that disturbance in sleep maintenance may have specific characteristics in this group. The association between difficulty maintaining sleep and type-2 DM was also previously demonstrated by a study of Western populations. ${ }^{17}$

As noted above, epidemiological studies of general populations have suggested that chronic insomnia may be a risk factor for developing depression. ${ }^{30,31}$ In the present cross-sectional study, although the causal relationships could not be clarified, higher PSQI-J was significantly associated with poor HRQOL and depression in both the type-2 DM group and the general population group. In addition, scores for PCS, MCS, and CES-D were at almost the same level for individuals without sleep disturbance/insomnia in the type-2 DM and the general population group.

It has been reported that poor sleep quality did not correlate with increased $\mathrm{HbA} 1 \mathrm{c}$ level in patients of type-2 $\mathrm{DM},{ }^{32,33}$ whereas high $\mathrm{HbA1c}$ was significantly associated with insomnia symptoms in a Japanese nonpatient male population. ${ }^{34}$ Thus, the relationship between HbAlc level and insomnia symptoms has been inconclusive. In the present study, the HbA1c value was not statistically different between the type-2 DM subgroups with and without sleep disturbance/insomnia, and logistic regression analyses did not find an association between $\mathrm{HbAlc}$ and sleep disturbance/insomnia. As the improvement in sleep problem in diabetic patients is not accompanied by reduction of $\mathrm{HbA} 1 \mathrm{c},{ }^{32}$ the level of $\mathrm{HbA} 1 \mathrm{c}$ may not be predictive of the severity of sleep disturbance/insomnia in type-2 DM patients. On the other hand, living alone, which was correlated to long-term insomnia, ${ }^{35}$ was associated with sleep disturbance/insomnia symptoms, suggesting that psychosocial distress or the home environment related to single life may be a cause of sleep disturbance/insomnia symptoms even in this disease population.

The cause of the type-2 DM group's greater difficulty with sleep maintenance could not be clarified in the present study. However, higher sympathetic nervous activity due to dysautonomia among type-2 DM patients s,37 $^{36}$ is likely to cause microarousals that may lead to poor sleep maintenance. ${ }^{38}$ In addition, we consider that sleep disturbance/insomnia 
symptoms worsen as the number of complications from diabetes increases. ${ }^{39}$ Another possible reason is the greater prevalence of obstructive sleep apnea (OSA), an important cause of sleep maintenance insomnia, ${ }^{40}$ in type- $2 \mathrm{DM}$ population than in the general population. ${ }^{41}$ Higher BMI, one of the critical risk factors for the development of OSA, was a significant factor associated with sleep disturbance/insomnia symptoms in the type-2 DM group. However, while these speculations may explain the higher likelihood of the occurrence of sleep maintenance insomnia in the type-2 DM group, none of the subitems of $\mathrm{C} 5$, including pain and uncomfortable breathing, was significantly different between the type-2 DM and general population groups.

There are some limitations in this study. First, we cannot identify the causal relationship between the presence of sleep disturbance/insomnia symptoms and associated factors in the present study. Second, since the sample of type-2 DM individuals was recruited from a single medical institute, there may be sampling bias. Third, it was possible that some individuals affected with DM or other diseases were potentially included in the general population group. This may be one of the main reasons why the mean total score of PSQI-J was relatively high in the general population group. Fourth, participants who had subjective sleep disturbance/insomnia in this study were not clinically diagnosed but allocated by higher score of the PSQI cutoff point. Objective sleep measures are necessary to make a definite diagnosis and to clarify the presence/absence of other sleep disorders causative for participants' subjective disturbance in sleep maintenance such as OSA or restless legs syndrome. Likewise, other subjective scales related to DM were not used in the current study.

\section{Conclusion}

Individuals with type-2 DM may be more likely to experience sleep disturbance/insomnia compared with the general population, and disturbance in sleep maintenance was a specific feature of insomnia symptoms in this sample. Sleep disturbance/insomnia symptoms were thought to have a considerable impact on daytime functioning even in type-2 DM individuals. Future prospective study is necessary to clarify the positive effects of interventions for sleep disturbance/ insomnia in type-2 DM population.

\section{Acknowledgment}

This work was supported by a grant from the Research Project on Development of Agricultural Products and Foods with Health-Promoting Benefits (NARO), Japan.

\section{Disclosure}

Yuichi Inoue has received lecture fees from Takeda Pharmaceutical, Eisai, MSD, Astellas Pharma, Otsuka Pharmaceutical, Philips Respironics, and Yoshitomiyakuhin. The other authors report no conflicts of interest in this work.

\section{References}

1. Ettaro L, Songer TJ, Zhang P, Engelgau MM. Cost-of-illness studies in diabetes mellitus. Pharmacoeconomies. 2004;22:149-164.

2. De Groot M, Anderson R, Freedland KE, Clouse RE, Lustman PJ Association of depression and diabetes complications: a meta-analysis. Psychosom Med. 2001;63:619-630.

3. Vgontzas AN, Tan TL, Bixler EO, Martin LF, Shubert D, Kales A. Sleep apnea and sleep disruption in obese patients. Arch Intern Med. 1994;154:1705-1711.

4. Cappuccio FP, D’Elia L, Strazzullo P, Miller MA. Quantity and quality of sleep and incidence of type 2 diabetes: a systematic review and meta-analysis. Diabetes Care. 2010;33:414-420.

5. Skomro RP, Ludwig S, Salamon E, Kryger MH. Sleep complaints and restless legs syndrome adult type 2 diabetes. Sleep Med. 2001;2: 417-422.

6. Shoji S, Mukai T, Uchimura N. Sleep disturbance in the diabetic patients. Prog Med. 2004;24:987-992.

7. Nilsson PM, Roost M, Engstrom G, Hedblad B, Berglund G. Incidence of diabetes in middle-aged men is related to sleep disturbances. Diabetes Care. 2004;27:2464-2469.

8. Shan Z, Ma H, Xie M, et al. Sleep duration and risk of type 2 diabetes: a meta-analysis of prospective studies. Diabetes Care. 2015;38: 529-537.

9. Spiegel K, Tasali E, Leproult R, Van Cauter E. Effects of poor and short sleep on glucose metabolism and obesity risk. Nat Rev Endocrinol. 2009;5:253-261.

10. Twig G, Shina A, Afek A, et al. Sleep quality and risk of diabetes and coronary artery disease among young men. Acta Diabetologica. 2016;53:261-270.

11. Gonzalez-Ortiz M, Martinez-Abundis E, Balcazar-Munos BR, PascoeGonzález S. Effect of sleep deprivation on insulin sensitivity and cortisol concentration in healthy subjects. Diabetes Nutr Metab. 2000;13: 80-83.

12. Spiegel K, Leproult R, Cauter EV. Impact of sleep debt on metabolic and endocrine function. Lancet. 1999;354:1435-1439.

13. Tasali E, Leproult R, Ehrmann DA, Van Cauter E. Slow-wave sleep and the risk of type 2 diabetes in humans. PNAS. 2008;105:1044-1049.

14. Arora T, Chen MZ, Cooper AR, Andrews RC, Taheri S. The impact of sleep debt on excess adiposity and insulin sensitivity in patients with early type 2 diabetes mellitus. J Clin Sleep Med. 2016;12:673-680.

15. Møller JB, Pedersen M, Tanaka H, et al. Body composition is the main determinant for the difference in type 2 diabetes pathophysiology between Japanese and Caucasians. Diabetes Care. 2014;37:796-804.

16. Sridhar GR, Madhu K. Prevalence of sleep disturbances in diabetes mellitus. Diabetes Res Clin Pract. 1994;23:183-186.

17. Meisinger C, Heier M, Loewel H. Sleep disturbance as a predictor of type 2 diabetes mellitus in men and women from the general population. Diabetologia. 2005;48:235-241.

18. Carotenuto M, Bruni O, Santoro N, Del Giudice EM, Perrone L, Pascotto A. Waist circumference predicts the occurrence of sleepdisordered breathing in obese children and adolescents: a questionnairebased study. Sleep Med. 2006;7:357-361.

19. Carotenuto M, Santoro N, Grandone A, et al. The insulin gene variable number of tandem repeats (INS VNTR) genotype and sleep disordered breathing in childhood obesity. J Endocrinol Invest. 2009;32:752-755.

20. Kawakami N, Takatsuka N, Shimizu H. Sleep disturbance and onset of type 2 diabetes. Diabetes Care. 2004;27:282-283. 
21. Yagi A, Nishino Y, Ugi S, et al. The role of sleep disturbance and depression in patients with type 2 diabetes. Diabetes Int. 2011;2: 79-85.

22. Doi Y, Minowa M, Uchiyama M, et al. Psychometric assessment of subjective sleep quality using the Japanese version of the Pittsburgh Sleep Quality Index (PSQI-J) in psychiatric disordered and control subjects. Psychiatry Res. 2000;97:165-172.

23. Buysse DJ, Reynolds 3rd CF, Monk TH, Berman SR, Kupfer DJ. The Pittsburgh Sleep Quality Index: a new instrument for psychiatric practice and research. Psychiatry Res. 1989;28:193-213.

24. Komada Y, Nomura T, Kusumi M, et al. A two-year follow-up study on the symptoms of sleep disturbances/insomnia and their effects on daytime functioning. Sleep Med. 2012;13:1115-1121.

25. Kinoshita E. Examining 16 items shortened version CES-D scale. Natl Fam Res Jpn. 2001;2:141-154.

26. Poulin C, Hand D, Boudreau B. Validity of a 12-items version of the CES-D used in the National Longitudinal Study of Children and Youth. Chronic Dis Can. 2005;26:65-72.

27. Tokuda Y, Okubo T, Ohde S, et al. Assessing items on the SF-8 Japanese version for health-related quality of life: a psychometric analysis based on the nominal categories model of item response theory. Value Health. 2009; $12: 568-573$.

28. Ware JE, Kosinski M. Interpreting SF-36 summary health measures: a response. Qual Life Res. 2001;10:405-413.

29. Lefante Jr JJ, Harmon GN, Ashby KM, Barnard D, Webber LS. Use of the SF-8 to assess health-related quality of life for a chronically ill, low-income population participants in the Central Louisiana Medication Access Program (CMAP). Qual Life Res. 2005;14:665-673.

30. Nouwen A, Winkley K, Twisk J, et al. Type 2 diabetes mellitus as a risk factor for the onset of depression: a systematic review and metaanalysis. Diabetologia. 2010;53:2480-2486.
31. Fernandez-Mendoza J, Shea S, Vgontzas AN, Calhoun SL, Liao D, Bixler EO. Insomnia and incident depression: role of objective sleep duration and natural history. J Sleep Res. 2015;24:390-398.

32. Garfinkel D, Zorin M, Wainstein J, Matas Z, Laudon M, Zisapel N. Efficacy and safety of prolonged-release melatonin in insomnia patients with diabetes: a randomized, double-blind, crossover study. Diabetes Metab Syndr Obes: Targets and Therapy. 2011;4:307-313.

33. Nakajima H, Kaneita Y, Yokoyama E, et al. Association between sleep duration and hemoglobin A 1c level. Sleep Med. 2008;9:745-752.

34. Kachi Y, Nakao M, Takeuchi T, Yano E. Association between insomnia symptoms and hemoglobin A 1c level in Japanese Men. PloS One. 2011;6:e21420.

35. Ishigooka J, Suzuki M, Isawa S, Muraoka H, Murasaki M, Okawa M. Epidemiological study on sleep habits and insomnia of new outpatients visiting general hospitals in Japan. Psychiatry Clin Neurosci. 1999; 53:515-522.

36. Surwit RS, Schneider MS. Role of stress in the etiology and treatment diabetes mellitus. Psychosom Med. 1993;55:380-393.

37. Nielsen FS, Hansen HP, Jacobsen P, et al. Increased sympathetic activity during sleep and nocturnal hypertension in type 2 diabetic patients with diabetic nephropathy. Diabet Med. 1999;16:555-562.

38. Bonnet MH, Arand DL. Heart rate variability: sleep stage, time of night, and arousal influences. Electroencephalogr Clin Neurophysiol. 1997;102:390-396.

39. Foley D, Ancoli-Israel S, Britz P, Walsh J. Sleep disturbances and chronic disease in older adults results of the 2003 National Sleep Foundation Sleep in America Survey. J Psychosom Res. 2004;56:497-502.

40. Chung KF. Insomnia subtypes and their relationships to daytime sleepiness in patients with objective sleep apnea. Respiration. 2005;72:460-465.

41. West SD, Nicoll DJ, Stradling JR. Prevalence of obstructive sleep apnoea in men with type 2 diabetes. Thorax. 2006;61:945-950.
Neuropsychiatric Disease and Treatment

\section{Publish your work in this journal}

Neuropsychiatric Disease and Treatment is an international, peerreviewed journal of clinical therapeutics and pharmacology focusing on concise rapid reporting of clinical or pre-clinical studies on a range of neuropsychiatric and neurological disorders. This journal is indexed on PubMed Central, the 'PsycINFO' database and CAS,

\section{Dovepress}

and is the official journal of The International Neuropsychiatric Association (INA). The manuscript management system is completely online and includes a very quick and fair peer-review system, which is all easy to use. Visit http://www.dovepress.com/testimonials.php to read real quotes from published authors. 